\title{
APPLICATION OF NORMALIZED CROSS CORRELATION TO IMAGE REGISTRATION
}

\author{
Y. Raghavender Rao ${ }^{1}$, Nikhil Prathapani ${ }^{2}$, E.Nagabhooshanam ${ }^{3}$ \\ ${ }^{1}$ Associate Professor, Department of ECE, JNTUH College of Engineering, Kondagattu, Karimnagar, A.P., India \\ ${ }^{2}$ Student Member, IEEE, Department of ECE, JNTUH College of Engineering, Kondagattu, Karimnagar, A.P., India \\ ${ }^{3}$ Professor and Head, Department of ECE, Sridevi Women's Engineering College, Hyderabad, A.P., India
}

\begin{abstract}
Image correspondence and registration techniques have gained popularity in recent times due to advancement of utilization in digital media and its storage. The main problem associated with image processing is when it is applied to fields like robotic vision and machine vision. The problem is due to clutter, i.e. the same frame with different objects has to be matched. Hence there has been need for efficient techniques of Image Registration. This led to development of feature extraction techniques and template matching techniques. The normalized cross correlation technique is one of them. A classical solution for matching two image patches is to use the cross-correlation coefficient. This works well if there is a lot of structure within the patches, but not so well if the patches are close to uniform. This means that some patches are matched with more confidence than others. By estimating this uncertainty more weight can be put on the confident matches than those that are more uncertain. All the simulations have been performed using MATLAB tool.
\end{abstract}

Keywords: - Normalized Cross Correlation, Image Correspondence, Robotic Vision and Image Registration.

$* * *$

\section{INTRODUCTION}

The correlation between two signals or simply the cross correlation is a standard tool for evaluating the degree to which two signals are similar. It is an elementary approach to match two image patches, for feature detection [1] as well as a component of more sophisticated techniques [2]. The technique has several advantages. The first advantage is that the cross correlation is equitably simple to compute. When used for matching a patch in a typical position in an image, Fourier methods can be used to compute the cross correlation fast. The second advantage is that cross correlation is independent of translations and scaling in the intensity domain. Thus it is fairly detached of lighting variations. There have been many works in literature which use crosscorrelation for matching [2] [3].

Normalized cross correlation (NCC) has been commonly used as a metric to evaluate the degree of similarity (or dissimilarity) between two compared images. The main advantage of the normalized cross correlation over the ordinary cross correlation is that it is less sensitive to linear changes in the amplitude of illumination in the two compared images. Furthermore, the Normalized Cross Correlation is confined in the range between -1 and 1 . The setting of detection threshold value is much simpler than the cross correlation. The Normalized Cross Correlation does not have a minimal frequency domain expression. It cannot be directly computed using the more efficient FFT (Fast Fourier Transform) in the spectral domain. Its computation time increases dramatically as the window size of the template gets corpulent.

\section{CROSS CORRELATION}

The cross-correlation of two real continuous functions, $\varphi_{\mathrm{xy}}$ is defined by

$$
\varphi_{x y}(t)=\int_{-\infty}^{\infty} x(\tau-t) y(\tau) d \tau
$$

If we compare it to convolution

$$
x(t) * y(t)=\int_{-\infty}^{\infty} x(\tau-t) y(\tau) d \tau
$$

We can see that the only difference is that for the cross correlation, one of the two functions is not reversed. Thus,

$$
\varphi_{x y}(t)=x(-t) * y(t
$$

Since the operation of time reversal is the same as taking the complex conjugate in the frequency domain, we can write

$$
{ }_{x y}=F T[\varphi \mid x y(t)]=X^{*}(f) Y(f)
$$

Unlike convolution, cross-correlation is not commutative but we can write

$$
\varphi_{x y}(t)=\varphi_{y x}(-t)
$$

You can show this by letting $\tau^{\prime}=\tau-\mathrm{t}$ 
In the discrete domain, the correlation of two real time series

$$
\mathrm{x}_{\mathrm{i}}, \mathrm{i}=0,1, \ldots, \mathrm{M}-1
$$

And

$$
\mathrm{y}_{\mathrm{j}}, \mathrm{j}=0,1, \ldots, \mathrm{N}-1
$$

Is by analogy to equation (1-1) given by

$$
\begin{gathered}
\varphi_{\mathrm{xy}, \mathrm{k}}=\sum_{j=\max (0, k)}^{\min (M-1+k, N-1)} x_{j-k}+y_{j} ; \\
\mathrm{k}=-(\mathrm{M}+1), \ldots, 0, \ldots,(\mathrm{N}-1)
\end{gathered}
$$

In MATLAB, cross-correlations are computed with the function 'xcorr' which works in the frequency domain. To obtain the discrete version of $\varphi_{x y}$ as defined by equation (1-6), one reverses the arguments (i.e., one calls $\operatorname{phi}_{\mathrm{xy}}=\mathrm{xcorr}(\mathrm{y}, \mathrm{x})$ ). xcorr also pads the end of the shorter input with zeros so that they are the same length. Since MATLAB cannot have zero or negative indexes the cross correlation sample with zero lag is the central element in the output vector. An alternate way of doing the cross correlation without padding with zeros is using the conv command $\left(\mathrm{phi}_{\mathrm{xy}}=\operatorname{conv}(\mathrm{y}, \mathrm{x}(\mathrm{end}:-1: 1))\right)$.

\section{NORMALIZED CROSS -CORRELATION}

In seismology, correlation is often used to search for similar signals that are repeated in a time series - this is known as matched filtering. Because the correlation of two high amplitude signals will tend to give big numbers, one cannot determine the similarity of two signals just by comparing the amplitude of their cross correlation.

The normalized correlation [4][5] for two time series can be defined as

$$
\varphi_{x y}^{\prime}(t)=\frac{\varphi_{x y}(t)}{\sqrt{\varphi_{x x}(0) \varphi_{y y}(0)}}
$$

The normalized quantity $\dot{\varphi}_{\mathrm{xy}}(\mathrm{t})$ will vary between -1 and 1 . A value of $\dot{\varphi}_{\mathrm{xy}}(\mathrm{t})=1$ indicates that at the alignment $\mathrm{t}$, the two time series have the exact same shape (the amplitudes may be different) while a value $\dot{\varphi}_{\mathrm{xy}}(\mathrm{t})=-1$ indicates that they have the same shape except that they have the opposite signs. A value of $\dot{\varphi}_{\mathrm{xy}}(\mathrm{t})=0$ shows that they are completely uncorrelated. In practice when one applies this normalization to real discrete signals, one will find that a correlation coefficient greater than about 0.7 or 0.8 indicates a pretty good match.

\section{APPLICATION OF NORMALIZATION TO IMAGE MATCHING}

If the two image regions are feeble variations of the same scene, then the heuristic [5] change has little effect. On the other hand, if there is a large difference in contrast between the matched regions, then the modified normalization potencies the correlation to zero (i.e. effectively uncorrelated).Finally, contrast normalization allows matching with regions that are principally uniform except for random or texture variations. This technique has numerous applications like MRI Image Section Matching and RGB Color Image Matching [7] [8].

\section{IMAGE REGISTRATION}

Image registration [9] essentially consists of following steps as per Zitova and Flusser [6].

- Feature detection: Salient and distinctive objects (closed-boundary regions, edges, contours, line intersections, corners, etc) in both reference and sensed images are detected.

- Feature matching: The correspondence between the features in the reference and sensed image established.

- Transform model estimation: The type and parameters of the so-called mapping functions, aligning the sensed image with the reference image, are estimated.

- Image resampling and transformation: The sensed image is transformed by means of the mapping functions.

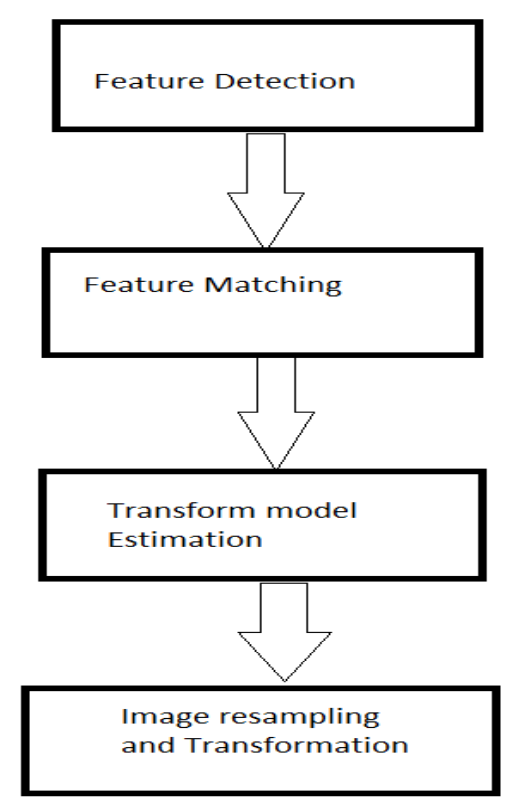

Fig.1. Steps involved in Image Registration 


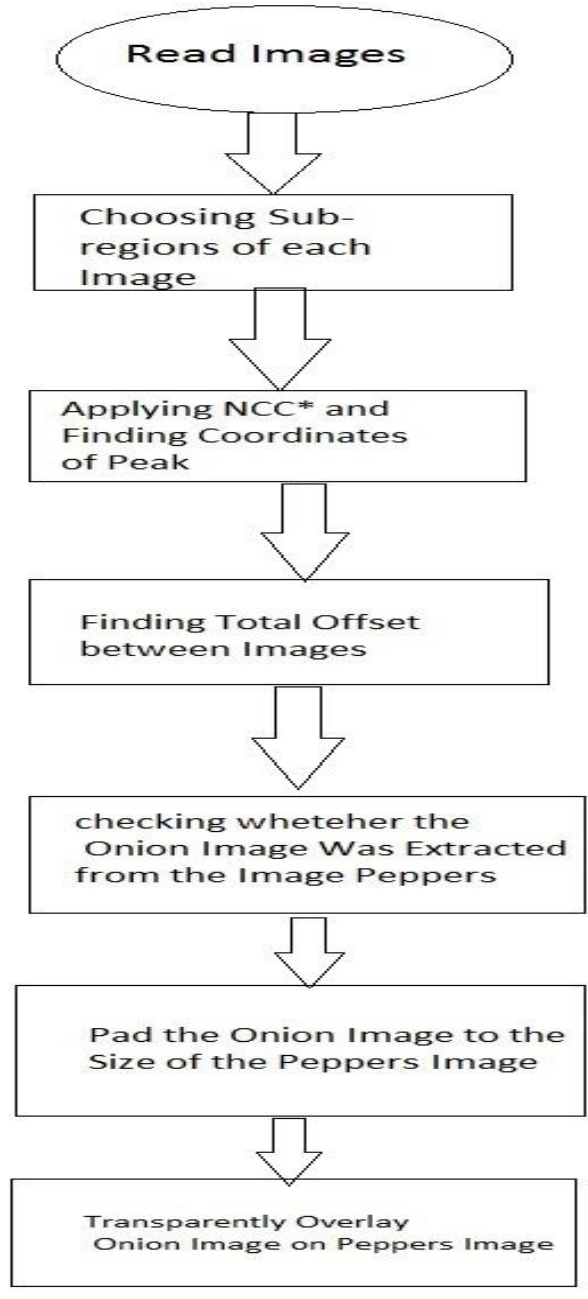

Fig.2. Pictorial Description of the Algorithm

\section{ANALYSIS AND RESULTS}

We consider the following two images for evaluation of the algorithm.

The "Onion Image" and the "Peppers Image".

Step 1: Reading the Images.

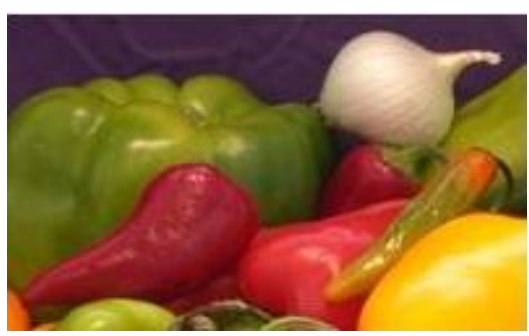

Fig.3. The Onion Image

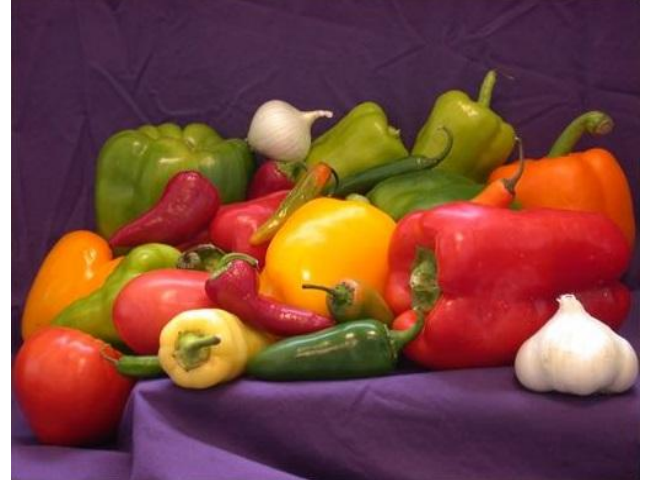

Fig.4 The Peppers Image

Step 2: Choose Subregions of Each Image.

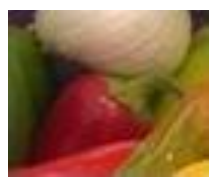

Fig.5 Subregion of the Onion Image

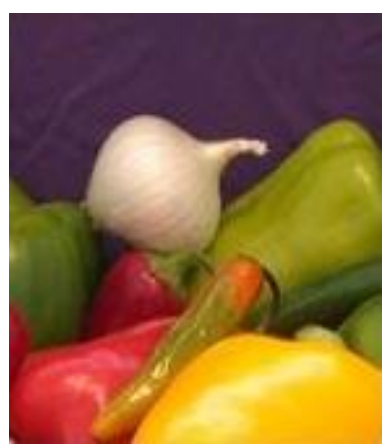

Fig.6 Subregion of the Peppers Image

Step 3: Performing Normalized Cross-Correlation and Finding Coordinates of Peak.

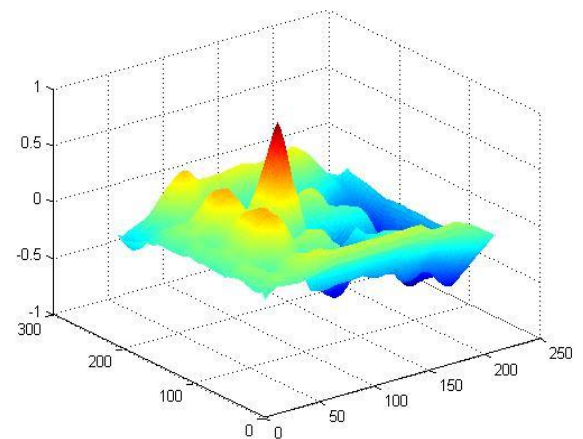

Fig.7 Coordinated plot of the registered image 
Step 4: Find the Total Offset Between the Images.

Step 5: Checking whether the Image Onion Was Extracted from the Image Peppers.

Step 6: Padding the Onion Image to the Size of the Peppers Image.

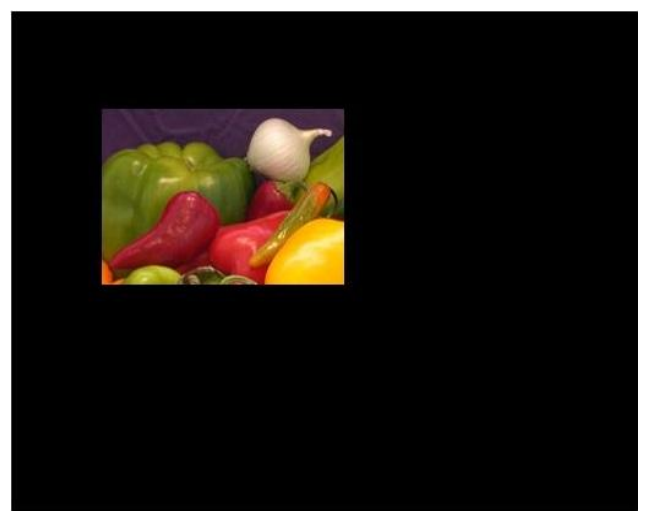

Fig.8 Padding Onion image to the size of Peppers image

Step 7: Transparently Overlay Onion Image on Peppers Image.

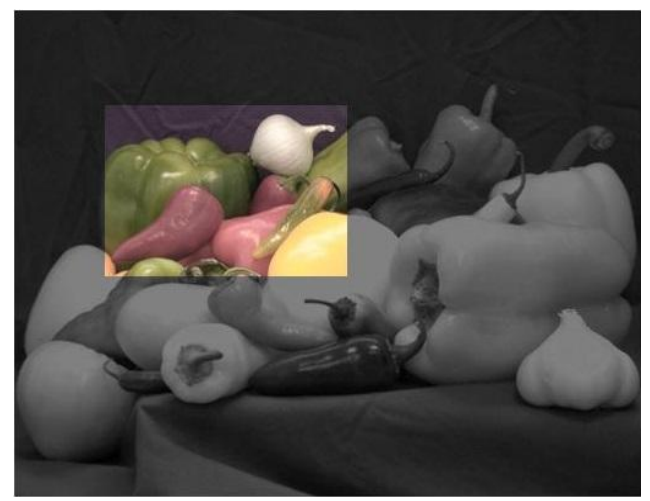

Fig.9. Transparent overlay of onion image onto Peppers image

\section{CONCLUSIONS}

Many other approaches to the image registration and correspondence problem are possible: this is one of the simplest. For example, features and their extraction techniques that are robust against various transformations can be found and matched for images from very different viewpoints; at the other extreme, parametric optic flow models can be fitted to spatial and temporal image gradients, offering high speed for images from close viewpoints. Finding correspondences remains a very active area of research. The Registration and correspondences of images in turn is based on cross correlation and normalized cross correlation techniques have been studied the least and they are an active area of research.
Over the last few years, image registration has been a very successful topic with multiplicity of applications. Registration is often a constituent of an image processing analysis package. Approaches like segmentation and quantification may be required to form a computer aided diagnostic system as a whole. Ensued by image registration in the medical applications, segmentation is needed to determine areas of interest in the image and in many cases accurate demarcation of objects vintages valuable information. Quantification is often the eventual goal principally in medical applications. Thus advancement in image registration has to go hand in hand with supplementary areas as well.

\section{REFERENCES}

[1]. R. O. Duda and P. E. Hart.Pattern Classification and Scene Analysis.Wiley-Interscience, 1973.

[2]. R. Brunelli and T. Poggio. Face recognition: Features versus templates. IEEE Trans. Pattern Analysis and Machine Intelligence, 15(10):1042-1052, 1993.

[3]. J. Sullivan, M. Blake, M. Isard, and J. MacCormick. Bayesian object localisation in images. International Journal of Computer Vision,44(2):111-135, 2001.

[4]. J.P. Lewis, "Fast Normalized Cross Correlation", Industrial Light and Magic.

[5]. Shou-Der Wei and Shang-Hong Lai," Fast template matching algorithm based on normalized cross correlation with adaptive multilevel winner update", IEEE Transactions on Image Processing, Vol. 17, No. 11, Nov. 2008.

[6]. Barbara Zitova, Jan Flusser, "Image registration methods: a survey", Image and Vision Computing 21 (2003), 977-1000

[7]. J. B. Antoine Maintz and Max A. Viergever, "ASurvey of Medical Image Registration", Medical Image Analysis (1998) Volume2, number 1, pp 1-36, Oxford University Press.

[8]. C. R. Maurer, G. B. Aboutanos, B. M. Dawant, R.A. Margolin, R. J. Maciunas and J. M. Fitzpatrick., "Registration of CT and MR brain images using a combination of points and surfaces", Medical imaging: image processing, volume 2434, Bellingham, WA, 1995. SPIE Press, 109-123.

[9]. Brown Gottesfeld L, "A survey of image Registration Techniques", ACM Computing surveys 24, 1992, 325-376.

[10]. Althof, R. J., Wind, M. G. J., \& Dobbins, J. T., III (1997). A rapid and automatic image registration algorithm with subpixel accuracy. IEEE Transactions on Medical Imaging, 16, 308-316.

[11]. Jignesh N Sarvaiya, Dr. Suprava Patnaik, and Salman Bombaywala, "Image Registration by Template Matching using Normalized Cross Correlation", International Conference on Advances Computing, Control, Telecommunication Technologies, pp. 819-822, 2009.

[12]. Minoru Mori and Kunio Kashino, "Fast Template Matching based on Normalized Cross Correlation using Adaptive Block Partitioning and Initial Threshold Estimation" IEEE International Symposium on Multimedia, pp. 196-203, 2010. 
[13]. Satoh, Shin'ichi; Katayama, Norio "An efficient evolution and implementation of robust face sequence matching", International conference on Image analysis and processing, pp. 266-271, 1999.

[14]. Barbara Zitova, Jan Flusser, "Image Registration Methods: a Survey", Image Vision Computing 21, PP. 9771000, 2003.

[15]. S. Damas, O. Cordon, and J. Santamaria, "Medical Image registration using Evolutionary Computation: An Experimental Survey", IEEE Computational Intelligence Magazine, Nov. 2011.

\section{BIOGRAPHIES:}

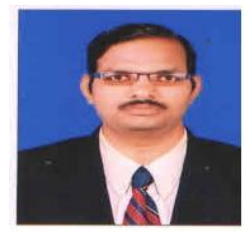

Y. Raghavender Rao is currently working as associate professor in ECE dept. at JNTUH College of Engineering., Kondagattu, Karimnagar, Andhra Pradesh, India. He has over fourteen years of teaching experience and his field of interest is Image processing. He has various publications in reputed International Journals.

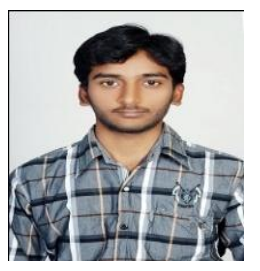

Nikhil Prathapani is an undergraduate Student of IV year - ECE at JNTUH College of Engineering., Kondagattu, Karimnagar, Andhra Pradesh, India. He has co-authored 2 papers in the field of Image Processing which have been published in reputed International Journals and presented 5 papers at various International conferences. His fields of interest include Image Processing, Wireless Networks' Simulation and Nano electronics.

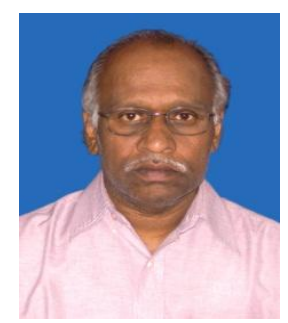

Dr. E. Nagabhooshanam is currently working as Professor and Head in ECE dept. at Sridevi Women's Engineering College, Hyderabad, Andhra Pradesh, India. He has 31 years of teaching experience. He has served in many important positions in highly esteemed colleges. He has published 14 papers in various International Journals and also International Conferences. His fields of interest include Control systems engineering and Image processing. 